\title{
Food Safety Practice and Its Associated Factors Among Food Handlers in Public Food Establishments of Godey Town, Somali Region, Eastern Ethiopia
}

\author{
Mohamed Mussema \\ (MPH) Public Health Officer
}

\begin{abstract}
Food borne illness is more common in both developed and developing country. In developing countries up to an estimated $70 \%$ of cases of diarrheal disease are associated with the Consumption of contaminated foods. Approximately 10 to $20 \%$ of food-borne disease outbreaks are due to contamination by the food handler. Nevertheless, data regarding food safety practice and associated factors in low-income countries like Ethiopia are very minimal, including the study area. The purpose of this study was to determine the magnitude of food safety practice and associated factors among food Handlers in public food establishments of Godey town, Somali region. To address the stated objective, institutional based cross sectional study design was used, on 390 food handlers working in all 109 public food establishments in Godey town. Data collection was done by four diploma nurse by using structured pretested questionnaire and institutional observation checklist. Data were entered on to Epi Data version 3.0 computer software and exported to SPSS version 20 computer software for analyzes. Accordingly, descriptive statistics was used to describe the independent variables in relation to the outcome variable. Bivariate, logistic regression analyses were done to see the association between each independent variable and the outcome variable. All variables with $\mathrm{p}$-value $<0.25$ during bivariate were entered in to multiple logistic regression models to control for all possible confounders and to identify factors associated with the outcome variable. Odds ratio along with $95 \%$ CI were estimated to measure the strength of the association. Finally, level of statistical significant was declared at $p$-value $<0.05$. As a result, the study has revealed that the prevalence of satisfactory food safety practice of food handlers was $20.9 \%$ [95\% CI: $16.7,24.8]$. In multivariable logistic regression analysis having knowledge about food safety [AOR $=10.4,95 \% \mathrm{CI}$ : $(4.6$, 23.81)], presence of hand washing facility for food handlers [AOR $=6.5,95 \% \mathrm{CI}:(2.7,15,3)]$ and presence of supervision $[\mathrm{AOR}=3.08,95 \% \mathrm{CI}:(1.3,7.12)]$ were statistically significant factors associated with food safety practice. Along with this, the prevalence of satisfactory food safety practice among food handlers was low in this study, knowledge about food safety, hand washing facility, and supervision were factors associated with food safety practice. Thus, strong supportive supervision, awareness on knowledge of food handlers on food safety practice and maintaining and establishing hand washing facility in kitchen area have to be done to solve the problem.
\end{abstract}

Kewwords: Ethiopia, food handlers, food safety practice, Godey, Somali

DOI: $10.7176 /$ RHSS/11-7-03

Publication date: April $30^{\text {th }} 2021$

\section{Introduction}

Safety is defined as the condition of being safe from undergoing or causing hurt, injury or loss (Webster's, 1990). Food safety was defined by the world health organization as assurance that when food is consumed in usual manner does not cause harm to human health and wellbeing (WHO, 2002).

Food is a fundamental human need and basic necessity to good health, Source of energy, vitamins, and minerals. If the hygienic condition of the food is not kept, it may cause an adverse effect on human health; this may result due to lack of knowledge, wrong attitude and improper practices by food producers or handlers at food catering establishments and consumers. Food safety still remains a major public health challenge and concern globally. Because of this Food borne diseases are known to be responsible for large proportion of illness, deaths and source of diarrheal diseases (Nagga Baraki, 2006).

Food can be subjected to contamination with toxic substances and pathogenic organisms during production, transportation, preparation, storage and service. The consumption of contaminated food that contains sufficient quantities of pathogenic organisms and toxic substances will result in food borne disease. It may due to lack of knowledge in part of food handlers and negligence in safe food handling. It is estimated that in developing countries up to $70 \%$ of cases of diarrheal disease may be caused by contaminated food. Food prepared in large quantity is liable to contamination and to the rise of food borne diseases if the strictest principles of hygiene are not maintained (WHO, 2006).

The study conducted by WHO in Geneva by the year 1989 showed that food handling personal play important role in ensuring food safety throughout the chain of food production and storage. Mishandling and disregard of hygienic measure on the part of food handlers may enable pathogenic bacteria to come in to contact 
with food and in some case survive and multiply in sufficient number to cause illness in consumers (WHO, 1989). Other evidence in University campus by the year 1988 indicated that poor sanitary practice in food distributions, storage, handling and preparation can create an environment in which bacteria and other infectious agent are more easily transmitted (Gent R. ,etal, 1999).

Food handler are anyone who works in a food and drink establishments and who handles food, or contact with any equipment or utensils that are likely to be in contact with food, such as cutlery, plates bowls, or chopping boards (Scallan E.et al, 2011).

Food borne diseases can be defined as diseases commonly transmitted through food. Food borne diseases comprise a broad group of illnesses caused by microbial pathogens, parasites; chemical contaminants and bio toxins (WHO, 2002).Food borne diseases are major health problems in developed and developing countries. The World Health Organization (WHO) estimated that in developed countries, up to $30 \%$ of the populations suffer from food borne diseases each year, whereas in developing countries up to 2 million deaths are estimated per year (Havelaar A. et al, 2013).

\section{Statement of the Problem}

Food-borne related illnesses have increased over the years, and negatively affected the health and economic well-being of many developing countries (WHO, 2008). The World Health Organization (WHO) stated that about 1.8 million persons died from diarrheal diseases in 2005, mainly due to the ingestion of contaminated food and drinking water.

Food poisoning can occurs as a result of consuming food contaminated with microorganisms or their toxins, the contamination arising from inadequate preservation methods, unhygienic handling practices, crosscontamination from food contact surfaces, or from persons harboring the microorganisms in their nares and on the skin (Barrie D., 1996).

According to CDC report, each year as many as 600 million, almost one in ten people in the world ill after consuming contaminated food, of this 420000 people die and from this, 125000 are under five, also CDC estimates that each year roughly 1 in 6 Americans (or 48 million people) gets sick, 128,000 are hospitalized, and 3,000 die of food borne diseases (CDC, 2001).According to WHO report 2009, say that fifty children under five die every hour in South East Asia due to diarrhea caused by food born disease (WHO, 2009).

Evidence indicates that in developing country Illness and death from diseases caused by contaminated food are a constant threat to public health and a significant impediment to socio-economic development worldwide. Food borne disease (FBD) outbreaks are common and often cause considerable morbidity and mortality (Havelaar A. et al, 2015).Including Ethiopia around 70\% of the diarrhea disease is associated with consumption of contaminated food (WHO, 2010). Approximately 10 to $20 \%$ of food-borne disease outbreaks are due to contamination by the food handler (Maizun M. and Nye N., 2002).

Practice of food safety among food handlers is not satisfactory in the world, even though it varies from country to country. For instance the study conducted in Turkey by the year 2004 on the evaluation of food hygiene knowledge, attitudes and practice of food handlers in food business among 764 food handler indicate that mean food safety practice score was only $43.4 \%+$ or -16.3 . The study demonstrated that food handlers in Turkish food businesses often have lack of knowledge regarding the basic food hygiene (critical temperatures of hot or cold ready-to-eat foods, acceptable refrigerator temperature ranges, and cross-contamination (Azmi Safak Ersun et al, 2004).

Other evidence indicate by the year between November 2010 and January 2011 in Switzerland on 100 food handlers in Restaurant showed that mean knowledge score of the participating food handlers was $71 \%$. None of the food handlers knew the correct temperatures for cooking chicken and holding potentially hazardous hot foods, and the range of temperatures for pathogen growth (Palak K.et al, 2013). The study conducted in Jamaica by 2014 , identified that factors like gender, education, job position, training and experience in the food industry were associated with the practice level of food handlers (Thelwell M., 2014).

Data regarding food borne diseases in African Region are still minimal (WHO, 2000). However, as per some studies conducted in the region, food safety practice of food handlers is not satisfactory. Study conducted in Nigeria by the year 2013 indicated that $37 \%$ of food handlers were directly engaged in cross contamination practices that are potential to cause food borne illness. Education and training on food safety were among the factors responsible for poor food preparation practice (Mokhtar A., 2013).Other evidence showed that lack of accesses to potable water; poor government structural arrangements and incontinent environmental condition were notable reason (Dewaal C. and Robert N., 2005).

In Ethiopia also the practice of food safety is not satisfactory with different factors. An evidence indicate that prevailing poor food handling and sanitation Practices, inadequate food safety laws, weak regulatory systems, lack of financial resources to invest safer equipment, and lack of education for food Handlers are the main contribution factor for food contaminations in public food catering establishments (Mulgeta $\mathrm{K}$ and Bahay A, 2012). Recent study conducted in Northern parts of Ethiopia in Gondar Town only 30.3\% of food handlers 
had good food handling practice and socio demographic characteristics, level of knowledge, attitude of food handler were identified factors for food safety practice( Gizaw Z. , et al, 2014).

Other study in Dangla Town in northern part of Ethiopia indicate that only 52.5\% of food handlers had good food handling practices and presence of insects and rodents were among the factors identified as having significant associations with food safety practice (Tessema G.et al, 2014).

Therefore conducting other study in order to strengthen the privies finding in areas where not previously done is very important. In Godey Town Somali Region there is no studies conducted on food safety practice and factors that shows the local context, regardless of some influencing factors. Those influencing factors include the existing large number of food establishments and food handlers serving large consumers as well as hot weather condition in the area. As per daily observations, the hot weather makes both cooked and non-cooked foods out of use easily unless safely handled. Therefore, this study was assess the status of food safety practice and associated factors among food handlers in public food establishments of Godey Town.

\section{Objectives of the Study \\ General objective of the Study}

The overall objective of the study was to determine the magnitude of food safety practice and associated factors among food handlers in public food establishments at Godey Town Somali Region.

\section{Specific objectives of the Study}

$>$ To estimate the magnitude of food safety practice among food handlers.

$>$ To identify factors associated with food safety practice among food handlers.

\section{Research Methodology}

In order to achieve the study objectives quantitative research approach was used. Specifically survey method was employed.

\section{Study Design}

Institutional based cross-sectional study design was used.

\section{Research Participants}

All food handlers who were working in Godey town public food establishments during study period. In this regard, while food handlers who were working in public food establishment during data collection were included from the study, food handlers who were unable to respond to interview during data collection were excluded in the study.

\section{Sample size determination}

The required sample size for this study was determined using single population formula with the following assumptions. Prevalence of satisfactory food safety practice to be $30.30 \%$ (Gizaw z. et al, 2014). It has 95\% confidence level, margin of error $\mathrm{d}=5 \%$ and $10 \%$ non-response rate, the sample size was calculated as follows. $\mathrm{n}=(\mathrm{z \alpha} / 2)^{2} \mathrm{p}(1-\mathrm{p})$ where $\mathrm{z} \alpha / 2$ at $95 \%$ confidence level $=1.96$ and $\mathrm{p}=0.303$ as given above. To this effect, the total sample size is $n=324$ and considering $10 \%$ for non-response $=356$.

In line with the above sample size determination logic, in Godey town there were 390 food handlers in public food establishments. As a result, the final sample size for this study was all food handlers who were working in public food establishments taken to increase the power of the study.

\section{Data collection methods \\ Data collection instrument}

The study has employed structured questioner to address the specific objectives of the study. The questioners contain socio demographic factor and other variables. The questioner was first prepared in English then translated in to Amharic and local language Somali then again translated to English by other person in order to check its appropriateness.

\section{Data Processing and Analysis}

Once the data was collected and cleaned, the data were entered into Epi data version 3.0 computer software and exported to SPSS version 20 computer software for analysis. Descriptive statistics was run to check for any missing values. Summary of descriptive statistics such as frequencies, percentages, means, standard deviation and median were used to describe socio demographic characteristics. Narration was used to report frequency of socio demographic characteristics of food handlers, Knowledge on Food Safety Practice of food handlers and attitude on Food Safety Practice of Food handlers. After that, the status of food safety knowledge, attitude and 
practice of food handlers were determined based on their score. To determine the status of knowledge based on 9 knowledge related question which contained three options, correct, incorrect and I don't know, the appropriate answer was given score one and the remaining given score of zero. The score was rang (0-9). The respondents who score 7 or $77 \%$ and above from total of nine where considered as adequate knowledge, food handlers score 6 and below 6 were considered as in adequate knowledge. food safety attitude of food handlers was assessed based on attitude related five question, correct answer was given score of 2, Neutral answer was given one, incorrect answer was given zero, where total range became (0-10).

After that respondents score range 6-10 were considered as positive attitude, range 4-5were considered as neutral attitude and rang 0-3 were considered as negative attitude,. Finally food safety practice was assessed based on 12 food safety practice related questions which contain three options always, sometimes and never. Correct answer was given 2, sometime was given one and incorrect answer was given zero. Total score was range from (0-24). Food handlers score 17 and above were considered as good, whereas food handlers score 16 and below 16 were considered as poor practice. During bivariate analysis, crude odds ratio along with $95 \%$ CI was used to see the association between each independent variable and the outcome variable by using binary logistic regression model. Independent variables with $\mathrm{p}$-value of $\leq 0.25$ were included in multi-variable analysis to control for all possible confounders and identify factors associated with food safety practice. Then outliers and influential cases were checked by standardized residuals and cook's distance respectively. Cases with standardized residuals out of the interval $(-3,3)$ and cook's distance above 1 were excluded from the multivariable analysis. Multi-co linearity was also checked to see the linear correlation among the independent variables by using standard error and variance inflation factor. Variables with standard error of $>2$ and variance inflation factor $>5$ were dropped from the multivariable analysis.

Hosmer Leme show goodness of fit tests was used to check for model fitness by looking cut of point $>0.05$. The continuous variables such as food handlers' age was tested using the normal curve with a histogram. Adjusted odd ratios along with $95 \%$ confidence interval was estimated to measure the strength of association between dependent and independent variable .In this study level of statistical significance was declared at $p$ value $<0.05$.

\section{Ethical Considerations}

The study protocol was approved by Haramaya University College of health and medical science intuitional health research and ethics review committee (IHRERC) of Harar campus. Official letter of cooperation was written from Haramaya University College of health and medical science to all concerned bodies of Godey town and Somali regional health bureau. The study participant ware clearly informed about the purpose of study. Benefit of the study was explained for the study participants. It was explained that the participation was voluntary. The issue of confidentiality and privacy of their information were clearly explained for the participants. The written informed voluntary and signed consent was obtained from the manager of the food establishment and each study participants. In order to protect the confidentiality of the information the name of participant was not included in the questioners. As a result, the identification was only coded by numerical numbers.

\section{Socio demographic characteristics}

A total of 390 food handlers in all (109) public food establishments were responded to the questionnaire yielding $98.2 \%$ response rate. Out of the total $(n=383)$ participant majority $140(36.6 \%)$ of study participants were from restaurant. Regarding gender, $(\mathrm{n}=383), 209$ (54.6\%) were males and $174(45.4 \%)$ were females. The mean age of the respondents was $26.4 \pm 8$ years. About their educational status majority of them $297,(77.5 \%)$ have no formal education. In the case of Ethnicity $276(72.1 \%)$ of the participants were Somali in their ethnicity. Looking marital status, around $282(73.6 \%)$ of participants were singles. In case of food safety training, three hundred five $(77.2 \%)$ of food handlers were not attend food safety training.

\section{Knowledge of food handlers about food safety}

Food safety knowledge of food handlers was assessed based on 9 food safety knowledge questions. Each question has three options (correct, incorrect and don't know). The appropriate answer for question number 6 and number 8 from the given option was incorrect, the remaining 7 questions, was option correct. Based on the result of knowledge assessments, from the total $(n=383)$ food handlers, $94(24.5 \%)$ food handlers had adequate knowledge and $289(75.5 \%)$ had inadequate knowledge. Mean score value of food safety knowledge of the respondents was 4.54 .

Attitude of food handlers towards food safety practice

Attitude on food safety practice of food handlers was assessed based on 5 attitude questions. From the total (383) study participants only 53(13.2\%) of food handlers had positive attitude. 


\section{Food safety practice among food handlers}

Current study indicated that only $80(20.9 \%)$ of the study participants had satisfactory food safety practice whereas 303 (79.1) of the study participants had not satisfactory food safety practice. However majority of the participants $225(58.7 \%)$ reported that always wash their hands before starting their work and $114(29.8 \%)$ of them do it sometimes.

The study also showed that only few of the participants $38(9.9 \%)$ they didn't use separate utensil when they are preparing raw and cooked foods. To ward checking expire date of product, only $33(8.6 \%)$ they never checked expire date of the products.

About smoking status, 103 (26.9\%) of participant reported that always smoke cigarette and $132(46.8 \%)$ of them smoke cigarette during normal work. From the total participant majority of them $300(78.3 \%)$ said that came to work when they have diarrhea. Furthermore $150(39.2 \%)$ participants reported that they always cover head during food serving and $210(54.8 \%)$ of the participants do it sometimes. Besides, majority 290 (75.7\%) participants reported that they have habit of making their nail long and 93 (24.3) of participants never do it. In addition from the total only few of them $35(9.1 \%)$ were reported that never disinfect cutting boards after each use.

\section{Observation of Institutional facilities}

Observation was conducted on 107 public food establishments 21 hotel, 39 restaurants, 26 cafeterias and 21 kurs bet. Two establishments were involuntary for participation (one from cafeteria, the other from kurs bet).Almost all $97(90.6 \%)$ had no food safety guideline. More than half $(78.5 \%)$ institution had no hand washing facility for food handlers.

Availability of guideline for food Establishments 10(9.3\%) 97(90.7\%) Presence of Hand washing facility. 23(21.5\%) 84(78.5\%) Availability of latrine. 56(52.3\%) 51(47.6\%) Availability of Insects/rodents 60(56\%) 47(44\%). Supervision held 19(17.8\%) 88(82.2\%).

\section{Factors Associated with food Safety Practice}

Identifying factors associated withfood safety practice was done using logistic regression model. After conducting univariate analysis to cheek data completeness and missing value of the data. Results of bivariate logistic regression analysis showed that factors like age group of 26- 36 year with [COR=12.333, 95\% CI: 6.391 , $23.80]$ primary, education with $[(\mathrm{COR}=0.11,95 \% \mathrm{CI}:(0.06-0.21))]$, secondary education with $(\mathrm{COR}=.086$, 95\% CI: $(0.036-0.2)]$, presence of food safety training with [(COR=12.36, 95\% CI (6.98-21.878)], having food safety knowledge with $[(\mathrm{COR}=15.71,95 \% \mathrm{CI}:(9.05-29.25)]$, availability of Food safety guideline with $(\mathrm{COR}=9.589,95 \% \mathrm{CI}:(2.34-37.8)]$, availability of Hand washing facility with $(\mathrm{COR}=7.6,95 \% \mathrm{CI}:(4.4-13.2)$ and supervision held with $[(\mathrm{COR}=14.1795 \mathrm{CI}:(7.18-25.5)]$, were statistically significant factors associated with food safety practice.

In the multivariable logistic regression analysis secondary education, having food safety knowledge, presence of hand washing facility for food handlers and presence of supervision were statistically significant factors food safety practice

\section{Discussion}

Current study showed that there was poor practice of food handling in the majority of food handlers 303(79.1). Factors associated with this poor finding were, lack of knowledge about food safety practice, lack of hand washing facility for food handlers and lack of supportive supervision from the regulatory body.

Looking the status of current study finding, the prevalence of good practice was only $20.9 \%$ this figure is markedly lower than the study conducted in Jordan (89.43\%) (Labib Sharif et al, 2013). This difference may be due to variation of socio demographic characteristics, regulatory system of food establishments and accesses difference in facility. However this study finding (20.9) was higher than the study conducted in Bangkok (15.2\%) (Cuprasitrut. T et al, 2011). This may due to difference in socio demographic characteristics and the number of sample size variation.

While looking knowledge about cross-contamination status of current study participants, $32.6 \%$ of food handlers reported that always come to work when they have diarrhea and $47.9 \%$ of participants do it sometimes, and only 18.2 of food handlers never wear jewelry during food preparation, these mentioned figures were not consistent with study conducted in Jamaica ,which showed that only $19 \%$ of food handlers came to work with illness like diarrhea, and $71 \%$ of food handlers were never use jewelry during food preparation. This possible variation may be due to; current study food handlers may have Lack of awareness on such type of risk activities to food born disease on food safety practice.

Finding of this study (good practice (20.9\%) also not consistent with study conducted in Sudan (33.21\%) (Abdelrazig A. et al, 2017). This difference may be due to study setting because; the study conducted in Sudan was by sample size of 40 food handlers and conducted in restaurants only. 
Current study also showed that the prevalence of good practice was lower than study conducted in Addis Ababa university cafeterias by the year 2014 on 302 food handlers (52.3\%), the study conducted in Arba Minch $32.6 \%$. This difference may be due to variation of geographical area and regulatory system. However the study conducted in ambo town on sanitary survey of food and drinking establishments, showed that the prevalence of not hand washing before starting their work was higher (5.7\%) (Dugassa Guteta, 2007) than current study (1.3\%) much lower $(1.1 \%)$ than smoking cigarette for current study $(27.7 \%)$. This possible reason may be due to hot environmental condition of current study area made food handlers better conscious to wash their hands to protract food spoilage and more cultural acceptance of smoking cigarette in current study area.

About knowledge on food safety practice, food handlers those who had good food safety knowledge on food safety practice were 10.4 times more good practice than those who had no adequate knowledge. Knowledge also as identified factor with the study conducted in, Kuching city. (Mizanur Rahmanet al, 2016). This possible explanation may be presence of knowledge about food safety enable/support food handlers to have satisfactory food safety practice.

While looking the other givens variables for the current study, presence of hand washing facility for food handlers also one of the identified factor, means that ,those who had hand washing facility in their kitchen area were 6.5 times more good practice than those who had not hand washing facility and when looking the other literatures this factor also as identified factors .for instance the study conducted in the study conducted in Mekele town (Lalit I. et al 2015), this possible reason may be that presences of hand washing facility may help the food handles to keep their personal hygiene like hand washing and to clean their utensil as time they wants,

Current study also identify that lack of supervision as gap for good safety practice, mean that those food handlers who were doing in facility where supervision held were 3 times had good safety practice than those who were doing in where there is not supervision held. This finding is supported with study conducted Cassino, Italy (Elisa Langianoet al, 2011) and in Arba Minch (Legesse D et al). Really the presence of supportive supervisors improve the food handlers, food handling practice by giving practical support and feedback on food handling.

\section{Conclusion and Recommendations Conclusion}

The magnitude of satisfactory food safety practice is low in this study. Variables like knowledge about food safety practice, hand washing facility for food handlers and presence of supervision were identified as factors significantly associated with food safety practice.

\section{Recommendations}

Based on major findings of the study important recommendations are listed below.

$>$ Godey Zonal as well as Godey warada Health bureau together with cultural and truism bureau is expected to work better in making strong supportive supervision for food establishments and it is better to Godey warada environmental health professions give awareness on food safety practice and food safety knowledge.

$>$ The local NGO should engage in awareness giving activities about food safety factors like food born disease and cross contamination on food born disease.

$>$ Godey warada health office in collaboration with water office, they should inform and support public food establishments in maintaining and establishing hand Washing facility in kitchen area to improve the overall sanitation of food establishment

\section{References}

Abdelrazig A, Mustafa K, Mohamed M. (2017). Hygienic Practices among food handlers in Restaurants of Al Nohod locality market west kordo fan Sudan-2017 inj pub health 2:129.

Abdolmajid Fadaei. (2015). Assessment of Knowledge, Attitudes and Practices of Food workers about food hygiene in Shahrekord Restaurants Iran World Applied Sciences Journal 33(7): 1113-1117.

Afolaranmi O.T, Hassan I.Z. Bello A.D., Misari.Z. (2015). Knowledge and practice of food Safety and hygiene among food vendors in primary schools in Jos, Plateau State, North centralNigeriaE3 Journal of Medical Research Vol. 4(2). 016-022,

Ab. Hamid, Rosnani ,Radu, Son, Othman, Mohhidin, Poh See, Toh, Lay Ching Cha. (2014). Assessment of Knowledge, Attitude and Practices Concerning Food Safety among Restaurant Workers in kualapilah, Malaysia j/Food Science and Quality Management32:2224-6088

Akabanda FHlortsi E. H. and Owusu-Kwarteng, J. (2017). Food safety knowledge, attitudes and practice of institutional food handlers in Ghana BMC Public Health, 17: 40-53 
Asrat M., Andualem, H., Worku,T., Tafesse,L. (2015).Assessment of the Sanitary Conditions of Catering Establishments and Food Safety Knowledge and Practices of Food handlers in Addis Ababa University Students' Cafeteria, Science Journal of Public health. 3(5):733-743.

Azmi Safak Ersun Gokhan Kivanc, Murat Bas. (2006). The evaluation of food hygiene knowledge, attitude and practice of food handlers in food businesses in Turkey, Journal of Food Control 17 : 317-322.

Barrie D. (1996) theprovision of food and catering services in hospital. J Hosp Infect. 33(1):13-33.

Centers for Disease Control and Prevention. (2001). CDC estimates of food-borne illnesses in the United States. Retrieved from http://www.cdc.gov /foodborne burden Retrieved from February 2015

Chigozie OIfeadike, Okechukwu CIronkwe, Prosper O. U.Adogu, Chinomnso nebue. (2014). Assessment of the food hygiene practices of food handlers in the federal Capital Territory of Nigeria tropical journal of medical research 17:1,10- 15

Cuprasitrut.T Srisorrachatr S.Mali D. (2011). Food safety knowledge, attitude and practice of food handlers in Rachathewi,Bangkok asia journal of public health 2:27-34.

Dewaal C., Robert N. (2005) Global and Local Food Safety around the World. Washington, D.C Center for Science in the Public Interest; 2005 doi:14.2046/j Foodcont.2005.12.007

Dugassa Guteta. (2007). Sanitary Survey of Food and Drinking Establishments in Ambo Town MPH Thesis, Ambo University, Ambo, Ethiopia. Retrieved from http://wjlsr.com/Date accessed January 2014.

Elisa Langiano, Maria Ferrara, Liana Lanni, Viviana Viscardi Angela Marie Abbatecola Elisabetta De. (2012). Vito Food safety at home: knowledge and practices of Consumers in Cassino, Italy J Public Health 20:4757 Evans HS, Madden P, Doudlas C, Adak G ObreinSj, Djuretic T Wall PG , StanwelL (2015 EU Summary report on zoonoses, zoonotic agents and food-borne outbreaks commundis public health 3:4551

Gent RN,TelphordD, Rand Syad Q. (1999). An outbreak of Campylobacter food poisoning University campus, 1999 commun dis public health 2:39-42

GizawZ, Gebrehiwot M, Teka Z. (2014). Food Safety Practice and Associated Factors of food Handlers Working in Substandard Food Establishments in Gondar Town Northwest Ethiopia. Int J Food Sci Nutr Diet 3: 138-14

Havelaar AH, Kirk M, Torgerson PR, Gibb HJ, HaldT, Lake RJ, et al(2015). Global Estimates and Regional comparisons of burdens of food born disease /journal.pmed.,12(12): e1001923

Kibret M, Abera B: The sanitary conditions of food service establishments and food safety Knowledge and practices of food handlers in Bahir Dar town. Ethiopian J Health Sci22 (1):27-35.

Labib Sharif, Mohammad M, Mohammad-Raed Al-Dalalah. (2013). Food hygiene Knowledge and practice of food handlers in military hospital Jordan journal of Food and Nutrition Sciences4, 245-251.

Lalit, I. Brkti, G. and Dejen,Y (2015). Magnitude of hygienic practices and its associated Factors of food handlers working in selected food and drinking establishments in Mekelle Northern Ethiopia international food research journal 22(6): 2650- 2656.

Legesse D, Tilahun M, Agedew E, Haftu D. (2017). Food Handling Practices and Associated Factors among Food Handlers in Arba Minch Town Public Food Establishments In Gamo Gofa Zone, Southern Ethiopia. J Epidemiology (Sunnyvale) 7: 302

Maizun MZ, Nyi NN. (2002). Socio demographic characteristics and KAP of food handlers towards Food sanitation in Malaysia South east Asianj j. trop. Med. and public health 33(2)12-33.

Melese Temesgen. (2015). Food Standards, Food Law and Regulation System in Ethiopia: A review Public Policy and Administration Research Vol.5, page 2224-5731

Mizanur Md and Rahman,mohd. (2016). Food safety practice among street food vendors in Northern Kuching city Sarawak 2016life science journa:7(2).

Mokhtar, A.A. (2013). Knowledge, attitudes, and practices of food service staff about food hygiene in hospitals in Makkaharea. Life Science Journal; 10(3).

Mulgeta K,and Bahay A. (2012). The sanitary condition of food service establishments 'food Safety Knowledge and practice of food handlers in Bahirdar town Ethiopia Healthsci22 (1): 27-35.

Nagga Baraki. (2006).food hygiene II for environmental health science student lecture note Series Haramaya university facility of health science in collaboration with the carter center EPHTI Addis Ababa 2006.

Noor Azira Abdul Mutalib, malinao, sman. (2012). Knowledge, attitude and practices regarding food hygiene and sanitation of food handlers in kualapilah Malaysia https:///doifood Count 2012

Oz FoodNet Working Group. (2007) Monitoring the incidence and causes of diseases potentially Transmitted by food in Australia: annual report of the Oz food net network, Commun Dis Intell Q Rep 31: 345-365.

Palak K. Panchal, Pierre Bonhote and Mark S.Dworkin. (2013). Food safety knowledge among Restaurant food handlers in Neuchâtel, Switzerland 2013, IA 50322- 2864 Vol 33, No. 3.p. 133-144.

Scallan E, Hoekstra RM, Angulo FJ, Tauxe RV, Widdowson M, Roy SL,et al. (2011). Food borne Illness Acquired in the United States Major Pathogens. Emerge Infect Dis.2011;17(1):7-15 
Sylvester N. O. and Craig, W. H. (2013). An Assessment of Food Safety Needs of Restaurants `in Owerri, Imo State, Nigeria. International Journal of Environmental Research and Public Health 10, 3296- 3309

Tessema, A. G., Gelaye, K. A., \&Chercos, D. H. (2014). Factors affecting food handling Practice among food handlers of Dangla town food and drink establishments North West Ethiopia BMC Public Health, 14,571.2458-14-571

Thelwell, M.R. (2014). Result. Food Safety Knowledge and Self-Reported Practices of Food Handlers in Jamaica,(unpublished doctoral dissertation), University of Walden.(Scholar works), Minneapolis, Minnesota. U.S.A.

T.Kuchenmüller S. Hird, C Stein, P Kramarz, A Nanda, A H Havelaar. (2009). WHO (2009) Estimates of the global burden of food borne diseasesJ. public health 14:18 Webster's Ninth New Collegiate Dictionary, 1990 web site www.worldcat.org/title/websters new-collegiate

WHO (World Health Organization). (2002). Global strategy for food safety: safer food for better Health Geneva Switzerland. Retrieved from http://www.who.int/fsf, Date accessed September 2002.

WHO. (2006) five keys to safer food manual Geneva 2006.Retrieved from http://www.who.int/iris/handl

WHO. (1989). Health surveillance and management procedure for food handling personal Geneva Retrieved from http://www.who.int/iris/handle Date Accessed April 1989 WHO (2009). National Food Control Systems and Consumer Participation in Food Standards Setting Codex Alimentarius Commission Rome, Italy

WHO. (2008). Food borne Disease: A focus for Health Education. Geneva Switzerland: WHO (1999-2000) Surveillance Program for Control of Food borne Infections and Intoxications In Europe, "8th Report WHO (2007). Food Safety and Foodborne Illness. Fact sheets No. 237. Geneva: Retrieved from https://foodhygiene Date accessed March 2007. 